1363). The present report is the first emphasizing the effectiveness of the ketogenic diet in infants. The attending adverse events and those reported in older children demonstrate the importance of close monitoring by a specialized medical team and support staff.

\title{
LOW-DOSE PYRIDOXINE MASKS PYRIDOXINE-DEPENDENT SEIZURES
}

A 4-month-old male infant with pyridoxine dependency and seizures from birth was controlled with extremely low-dose pyridoxine $(0.5 \mathrm{mg} /$ day $)$ given as a vitamin supplement, in a report from Joana de Gusmao Children's Hospital, Florianopolis, Brazil. Seizures were generalized myoclonic and tonic postures that began at 18 days of age and continued until 30 days. After a seizure-free period between 30 and 80 days, seizures returned and were refractory to antiepileptic drugs (AED) and prednisone. Examination showed global neurodevelopmental delay. An interictal EEG recorded continuous delta activity. MRI and laboratory tests were negative. Foliowing a diagnostic trial of pyridoxine $50 \mathrm{mg}$ twice daily by enteral tube, seizures ceased but the infant became comatose and hypotonic. After gradually regaining consciousness, the infant was discharged on $50 \mathrm{mg}$ pyridoxine orally, twice daily. Further history taking revealed that a multivitamin supplement containing $0.5 \mathrm{mg}$ pyridoxine daily had been given during the seizure-free interval between 30 and 80 days. An interruption in vitamin supplement coincided with the seizure relapse. At 2 years of age, the child had 2 brief seizures with fever. With an increase in pyridoxine dosage to 200 $\mathrm{mg} /$ day, no further seizures had occurred at last follow-up at 3 years, and development was normal. (Grillo E, da Silva RJM, Barbato JH Jr. Pyridoxinededpendent seizures responding to extremely low-dose pyridoxine. Dev Med Child Neurol June 2001;43:413-415). (Respond: Eugenio Grillo MD, Hospital Infantil Joana de Gusmao, Servico de Neurologia, Rua Rui Barbosa 152, 88025-301, Florianopolis SC, Brazil).

COMMENT. Low-dose pyridoxine given as a routine vitamin supplement may be sufficient to control seizures and mask an underlying pyridoxine-dependency. Early diagnosis and appropriate treatment may be delayed. In this case the masking dose of pyridoxine was very low, between 0.05 and $0,1 \mathrm{mg} / \mathrm{kg} / \mathrm{day}$.

Impaired consciousness and hypotonia have been reported in pyridoxine dependency after diagnostic doses given intravenously (Bankier et al, 1983), but rarely with enteral administration, as in this case. The associated irregular breathing was a concern, and further pyridoxine withdrawal and diagnostic testing were omitted.

Baxter P of the Sheffield Children's Hospital, UK, provides an excellent review of pyridoxine-dependent and -responsive seizures (Dev Med Child Neurol June 2001;43:416-420). Although rare, the varied modes of presentation of pyridoxine-dependent seizures result in misdiagnosis, particularly those with coincident hypoxic-ischemic encephalopathy, intraventricular hemorrhage, or hydrocephalus. Neuroimaging may show a variety of abnormalities, including paraventricular cysts, corpus callosum hypoplasia, cerebellar hypoplasia, white matter lesions, and hydrocephalus. Patients presenting after 1 month of age have a better outcome than those with earlier onset. Early and sustained treatment with adequate dosage influences outcome, especially in prevention or lessening of cognitive and developmental delays. With large doses of pyridoxine, monitoring of Romberg sign, ankle jerks, and joint position when possible is advised to check for sensory changes. There is no specific test for pyridoxine dependency, except for withdrawal and a further response to pyridoxine when seizures recur. As seen above, this test is not without hazard. Siblings and cousins may be affected and the 
disorder is genetic with a recessive inherited pattern. A site on chromosome 5 has been identified but not localized exactly.

Except for a recent report of elevated pipecolic acid, other biochemical theories including glutamic acid decarboxylase deficiency have not been confirmed. (See Ped Neur Briefs 2000;14:27; 2000;14:52). One report of hyperprolinemia type II in a girl aged 20 months with convulsions and encephalopathy responded to IV and subsequent oral pyridoxine. The proline metabolite that accumulates in this disorder inactivates vitamin B6.

\section{TRAUMATIC BRAIN DISORDERS}

\section{BRAIN DAMAGE FOLLOWING INFLICTED HEAD INJURY}

Detailed neuropathological studies, including immunocytochemistry for microscopic damage, are reported in 53 cases of non-accidental head injury in children examined by 2 neuropathologists at the Universities of London and Sheffield, UK. Only 7 of the series had admissions of child abuse. Thirty seven were infants, with age at injury ranging from 20 days to 9 months, and 16 children with injury sustained at 13 months to 8 years. Skull fractures were present in $36 \%$ of cases, usually parietal or occipital, acute subdural bleeding in $72 \%$, and retinal hemorrhages in $71 \%$. None had extradural hematoma. Raised intracranial pressure secondary to brain swelling was the cause of death in $82 \%$ of cases. Microscopic exam revealed signs of severe hypoxic brain damage in $77 \%$, vascular axonal damage in $40 \%$, and diffuse traumatic axonal damage in only $6 \%$. Localized axonal injury to the craniocervical junction of the spinal cord was present in 11 cases, all infants. Age-related patterns of damage were significant. Infants of 2-3 months presented with apnea and skull fracture, showed craniocervical axonal damage, a thin subdural hemorrhage, but no extracranial injury. Eight infants with no signs of impact were assumed to have "shaken baby syndrome." They had presented with collapse or respiratory arrest. The brain was swollen, 7 had a thin film of subdural hemorrhage, and 5 had bilateral retinal hemorrhages. The pathology in this "shaken-only" group was not different from the 29 infants with evidence of impact. Children over 1 year had a greater incidence of severe extracranial, especially abdominal, injuries, larger subdural hemorrhages, and adult patterns of hemispheric white matter damage. Diffuse axonal injury was an uncommon sequel of inflicted head injury in children. (Geddes JF, Hackshaw AK, Vowles GH, Nickols CD, Whitwell HL Neuropathology of inflicted head injury in children. I. Patterns of brain damage. Brain July 2001;124:1290-1298). (Respond: Dr JF Geddes, Department of Histopathology and Morbid Anatomy, Royal London Hospital, Whitechapel, London E1 1BB, UK).

COMMENT. Patterns of brain damage in inflicted head injury in children are different from adults and also vary with the age of the child. Extradural hematomas are rare, subdural hematomas are thin films not requiring neurosurgical intervention and never massive, and subarachnoid bleeding is also rarely clinically significant. Traumatic axonal damage is mainly focal, involving the lower brainstem and cervical roots, and only occasionally diffuse. Whereas infants have craniocervical axonal damage as a result of cervical hyperextension/flexion stretch injury ("shaken-bay syndrome"), children have a greater incidence of severe extracranial, abdominal injury, and larger subdural hematomas with non-accidental inflicted head injury.

The authors have followed this paper with part II. Microscopic brain injury in infants (Geddes JF et al. Brain July 2001;124:1299-1306). The findings corroborate those outlined above. 